\title{
Guest editorial for the IJCARS special issue on JAMIT 2015 and 2016
}

\author{
Akinobu Shimizu ${ }^{1} \cdot$ Yoshinobu Sato $^{2} \cdot$ Kiyonari Inamura $^{3}$
}

Published online: 8 April 2017

(c) CARS 2017

This special issue of the International Journal of Computer Assisted Radiology and Surgery (IJCARS) presents a selection of the works presented at the 34th and 35th annual conferences of the Japanese Society of Medical Imaging Technology (JAMIT). The 34th conference was held from 30 July to 1 August 2015 at the Kanazawa theatre in Kanazawa. The 35th annual conference was held from 21st to 23rd July 2016 at Keyaki Kaikan of Chiba University in Chiba, Japan. We are extremely grateful that we are able to deliver this special issue, which is based on the strong collaboration of IJCARS and JAMIT.

The main topics of the conferences were as follows:

- Medical imaging and reconstruction theory (CT, MR, X-ray, US, PET, SPECT, OCT, biological image, histological image, etc.).

- Medical image analysis (segmentation, registration, feature extraction, etc.).

Akinobu Shimizu

simiz@cc.tuat.ac.jp

http://web.tuat.ac.jp/ simizlab/

Yoshinobu Sato

yoshi@is.naist.jp

http://icb-lab.naist.jp/english/index.html

Kiyonari Inamura

ina-kiyo@mue.biglobe.ne.jp

1 Department of Electrical and Electronic Engineering, Institute of Engineering, Tokyo University of Agriculture and Technology, 2-24-16 Naka-cho, Koganei-shi, Tokyo 184-0012, Japan

2 Graduate School of Information Science, Nara Institute of Science and Technology (NAIST), 8916-5 Takayama-cho, Ikoma, Nara 630-0192, Japan

3 Osaka University, Onoharahigashi 6-35-7, Minoo-city, Osaka 562-0031, Japan
- Computational anatomy and computational physiology.

- Computer-aided diagnosis (CAD).

- Computer-aided surgery and therapy (medical robots, surgical and interventional systems, etc.).

- Visualization, interactions, virtual/augmented reality, and simulations.

- Medical informatics (PACS, IHE, telemedicine, e-health, etc.).

The conference attracts many engineers, clinicians, and scientists in Japan. In 2015, 46 oral presentations and 38 poster presentations were given, and 65 oral presentations and 52 poster presentations were provided in 2016. We encouraged authors of high-quality presentations of the JAMIT annual conferences to submit to the IJCARS JAMIT 2015-2016 special issue. Eventually, 31 papers were submitted for the special issue after extending the JAMIT conference proceedings to full papers to be considered for publication in IJCARS. All the submitted papers underwent the regular IJCARS review procedure to keep the paper quality sufficiently high.

The current special issue includes the following seven research papers, which are related to the first four topics among the seven topics of the conference. The first paper was presented by Sato et al. and relates to the topic, "medical imaging and reconstruction." It proposes a method for geometry calibration of a portable radiography device and demonstrates tomosynthesis image reconstruction by using the proposed method. Three papers relate to both "medical image analysis" and "computational anatomy and computational physiology." Hanaoka et al. presented an algorithm for automated detection of anatomical anomaly in terms of the number of vertebral bones based on CT volume. Fukuda et al. proposed an approach for estimating patient-specific muscle attachment regions from 3-D CT images and validated it with 
cadaver experiments. Saito et al. attempted joint optimization for segmentation and shape priors, including translation, and demonstrated its effectiveness in gallbladder segmentation from a non-contrast $\mathrm{CT}$ volume. The remaining three papers were related to computer-aided diagnosis (CAD). Misawa et al. compared the diagnostic ability and efficacy of CAD for endocytoscopy with the performances of human experts and trainee endoscopists. Kawagishi et al. reported a comparative study of CAD for pulmonary nodule on CT images between classification accuracies using calculated image features and imaging findings annotated by radiologists. Takeda et al. verified the accuracy of the proposed normal model for FDG-PET and the usefulness of the statistical image analysis method by using typical liver, lung, and abdominal cancers.

A few papers are still under consideration for publication in a regular issue.

We trust that this special issue provides a snapshot of the best works in the JAMIT annual conferences in 2015 and 2016. We hope that the readers will enjoy this special issue.

We thank all the reviewers and the Editor-in-Chief, Prof. Heinz Lemke, of IJCARS for their continued support and contributions to the publication of a selection of papers from the JAMIT conferences. 